\title{
Coated and Hollow Microneedle-Mediated Intradermal Immunization in Mice with Diphtheria Toxoid Loaded Mesoporous Silica Nanoparticles
}

\author{
Guangsheng Du ' (D) - Laura Woythe' • Koen van der Maaden ' • Mara Leone' • Stefan Romeijn ' • \\ Alexander $\mathrm{Kros}^{2} \cdot$ Gideon Kersten ${ }^{1,3} \cdot$ - Wim Jiskoot ${ }^{\prime}$ Joke A. Bouwstra
}

Received: 29 May 2018 / Accepted: 6 August 2018 / Published online: 13 August 2018

(C) The Author(s) 2018

\begin{abstract}
Purpose To examine the immunogenicity of diphtheria toxoid (DT) loaded mesoporous silica nanoparticles (MSNs) after coated and hollow microneedle-mediated intradermal immunization in mice.
\end{abstract}

Methods DT was loaded into MSNs and the nanoparticle surface was coated with a lipid bilayer (LB-MSN-DT). To prepare coated microneedles, alternating layers of negatively charged LB-MSN-DT and positively charged N-trimethyl chitosan (TMC) were coated onto $\mathrm{pH}$-sensitive microneedle arrays via a layer-by-layer approach. Microneedle arrays coated with 5 or 3 layers of LB-MSN-DT were used to immunize mice and the elicited antibody responses were compared with those induced by hollow microneedle-injected liquid formulation of LB-MSN-DT. Liquid DT formulation with and without TMC (DT/TMC) injected by a hollow microneedle were used as controls.

Results LB-MSN-DT had an average size of about $670 \mathrm{~nm}$ and a zeta potential of $-35 \mathrm{mV}$. The encapsulation efficiency of DT in the nanoparticles was 77\%. The amount of nanoencapsulated DT coated onto the microneedle array increased linearly with increasing number of the coating layers. Nano-encapsulated DT induced stronger immune responses than DT solution when delivered intradermally via hollow microneedles, but not when delivered via coated microneedles.

Joke A. Bouwstra

bouwstra@lacdr.leidenuniv.nl

Division of BioTherapeutics, Leiden Academic Centre for Drug Research, Leiden University, Leiden, The Netherlands

2 Department of Supramolecular \& Biomaterials Chemistry, Leiden Institute of Chemistry, Leiden University, Leiden, The Netherlands

3 Institute for Translational Vaccinology (Intravacc), Bilthoven, The Netherlands
Conclusion Both the nano-encapsulation of DT and the type of microneedles affect the immunogenicity of the antigen.

KEY WORDS coated microneedles · diphtheria toxoid . hollow microneedles · intradermal vaccination · mesoporous silica nanoparticles

$\begin{array}{ll}\text { ABBREVIATIONS } \\ \text { APCs } & \text { Antigen-presenting cells } \\ \text { CLSM } & \text { Confocal laser scanning microscopy } \\ \text { DLS } & \text { Dynamic light scattering } \\ \text { DOPC } & \text { 1,2-dioleoyl-sn-glycero-3-phosphocholine } \\ \text { DOPS } & \text { 1,2-dioleoyl-sn-glycero-3-[phospho-L- } \\ & \text { serine](sodium salt) } \\ \text { DT } & \text { Diphtheria toxoid } \\ \text { EE } & \text { Encapsulation efficiency } \\ \text { LB-MSN-DT } & \text { DT loaded and lipid fused MSNs } \\ \text { LC } & \text { Loading capacity } \\ \text { MSNs } & \text { Mesoporous silica nanoparticles } \\ \text { PB } & \text { Phosphate buffer } \\ \text { PBS } & \text { Phosphate buffered saline } \\ \text { PDI } & \text { Polydispersity index } \\ \text { SEM } & \text { Scanning electronic microscopy } \\ \text { TMB } & \text { 3,3',5,5'-tetramethylbenzidine } \\ \text { TMC } & \text { N-trimethyl chitosan }\end{array}$

\section{INTRODUCTION}

Vaccination is one of the most cost-effective tools to prevent infectious diseases in human beings (1). Traditional vaccines are based on attenuated or inactivated pathogens. Nowadays, subunit vaccines containing only immunogenic parts of a pathogen are being extensively investigated because they are safer (2). The disadvantage of subunit vaccines is that they are generally less immunogenic than traditional vaccines. To 
overcome this, adjuvants such as immune modulators and nanoparticulate delivery systems can be used $(3,4)$.

Nanoparticles have been extensively studied for the delivery of vaccines, as they can improve the immunogenicity of antigens by enhancing the targeting of antigens to antigenpresenting cells (APGs) (5). Furthermore, the immune responses can potentially be modified by tuning the properties of nanoparticles such as size, surface charge, and release kinetics of antigens $(3,6,7)$. Among different types of nanoparticles, mesoporous silica nanoparticles (MSNs) have gained increasing attention because of their excellent biocompatibility and stability. Besides, the silica surface can be easily modified and functionalized and the large pores and surface area of MSNs enable efficient loading of antigens with a high loading capacity $(8,9)$. Studies have shown that antigen loaded MSNs are able to increase the uptake of antigens by APCs and improve immune responses in mice (9-11).

Vaccines are mostly administered by intramuscular or subcutaneous injection, but these methods have disadvantages such as low acceptance by a considerable number of people and infection risk due to needlestick injuries or reuse of needles (12-14). Additionally, the delivery of vaccines to APCs may be inefficient as these delivery sites are not rich of APCs (15). To avoid the drawbacks of hypodermic needles, microneedles have been developed. Microneedles are micrometer-sized needle-like structures and can be used to penetrate skin and deliver the antigen in a minimal invasive and pain-free way (16). The skin contains a large number of APCs, and therefore microneedle-mediated intradermal delivery of vaccines has potential for effective vaccination (17).

Several types of microneedles are in development, such as coated, dissolvable and hollow microneedles (16). On the one hand, coated and dissolvable microneedles are used to administer dry-state vaccine formulations (18), which offer the potential advantage of improving antigen stability $(16,19)$. Previously, silicon microneedle arrays with a $\mathrm{pH}$-sensitive surface were developed to bind negatively charged vaccines at slightly acidic conditions ( $\mathrm{pH} 5.8$ ) and release the coated material at physiological pH (7.4) (20). Several studies have shown that the antigen coated microneedles induced a similar immune response as subcutaneously or intramuscularly injected antigen solution (21-23). On the other hand, hollow microneedles are used to inject liquid formulations and the dose can be precisely controlled. We previously showed that hollow microneedles together with an applicator can be used to deliver antigen-loaded nanoparticles intradermally (24).

In this study, we aimed to examine the immunogenicity of intradermally delivered DT loaded MSNs by using either coated microneedle arrays or a single hollow microneedle. The microneedle arrays were coated with DT loaded in MSNs by using a layer-by-layer coating approach after which the delivered dose into ex vivo human skin was examined. In a subsequent immunization study, the antibody response induced by LB-MSN-DT coated microneedles was compared with that obtained after injection of a suspension of LB-MSNDT by hollow microneedles into mouse skin.

\section{MATERIALS AND METHODS}

\section{Materials}

DT (batch 04-44, $1 \mu \mathrm{g}$ equal to $0.3 \mathrm{Lf}$ ) and diphtheria toxin were provided by Intravacc (Bilthoven, The Netherlands). (3aminopropyl)triethoxysilane (APTES, 99\%), 4pyridinecarboxaldehyde $(97 \%)$, sodium cyanoborohydride ( $\left.\mathrm{NaBH}_{3} \mathrm{CN}, 95 \%\right)$, cholesterol $(\geq 99 \%)$, fetal bovine serum (FBS), M199 medium (with Hank's salts and L-glutamine) and bovine serum albumin (BSA) were obtained from Sigma-Aldrich (Zwijndrecht, The Netherlands). 1,2-dioleoylsn-glycero-3-phosphocholine (DOPG) and 1,2-dioleoyl-snglycero-3-[phospho-L-serine](sodium salt) (DOPS) were purchased from Avanti Polar Lipids Inc. (Alabaster, AL). Hydrogen peroxide $(30 \%)$ was purchased from Fluka (Steinheim, Germany). Toluene $(\geq 99.7 \%$ ) was obtained from Biosolve (Valkenswaard, The Netherlands). N-trimethyl chitosan (TMC) and rhodamine labeled TMC (TMC-Rho) were prepared as reported previously $(23,25)$. Glucose solution, Lglutamine $(200 \mathrm{nM})$, penicillin-streptomycin $(10,000 \mathrm{U} / \mathrm{mL})$ and 1 -step ${ }^{\mathrm{TM}}$ ultra 3,3',5,5'-tetramethylbenzidine (TMB) were purchased from Thermo-Fisher Scientific (Waltham, MA). IRDye 800CW protein labeling kit (low molecular weight) was ordered from LI-COR (Lincoln, NE). HRP-conjugated goat anti-mouse total IgG, IgG1 and IgG2a were ordered from Southern Biotech (Birmingham, AL). Sulfuric acid (95$98 \%$ ) was obtained from JT Baker (Deventer, The Netherlands). Sterile phosphate buffered saline (PBS, $163.9 \mathrm{mM} \mathrm{Na}^{+}, 140.3 \mathrm{mM} \mathrm{Cl}^{-}, 8.7 \mathrm{mM} \mathrm{HPO}_{4}{ }^{2-}, 1.8 \mathrm{mM}$ $\mathrm{H}_{2} \mathrm{PO}^{4-}$, $\mathrm{pH}$ 7.4) was ordered from B. Braun (Oss, The Netherlands). $1 \mathrm{mM}$ phosphate buffer $(\mathrm{PB})$ with a $\mathrm{pH}$ of 7.4 or 5.8 was prepared in the lab. Milli-Q water (18 M $\Omega / \mathrm{cm}$, Millipore Co.) was used for the preparation of all solutions. All the other chemicals used were of analytical grade.

\section{Preparation of DT Encapsulated and Lipid Fused MSNs (LB-MSN-DT)}

Plain MSNs with a particle size of about $200 \mathrm{~nm}$ and large pores (about $10 \mathrm{~nm}$ in diameter) were prepared and modified with amino groups to generate a positively charged surface, as described earlier $(11,26)$. To improve the colloidal stability of MSNs, liposomes were coated onto the surface of MSNs by using a method as previously described (11). These liposomes were prepared by lipid film hydration followed by sonication. Briefly, DOPG, DOPS and cholesterol with a molar ratio of 
7:1:2 were dissolved in chloroform in a round bottom flask. The organic solvent was evaporated by using a rotary evaporator (Buchi rotavapor R210, Flawil, Switzerland) for $30 \mathrm{~min}$. Subsequently, the lipid film was hydrated with $1 \mathrm{mM} \mathrm{PB}$ (pH 7.4) and vortexed for $10 \mathrm{~s}$ to form a lipid vesicle suspension. The suspension was sonicated in a Branson 2510 water bath (Danbury, CT) for $10 \mathrm{~min}$. The obtained liposomes were stored at $4^{\circ} \mathrm{C}$ in the refrigerator for further use.

To prepare LB-MSN-DT, $0.5 \mathrm{~mL}$ MSNs (2 mg/mL) and $0.5 \mathrm{~mL}$ DT $(0.5 \mathrm{mg} / \mathrm{mL})$ were mixed in $1 \mathrm{mM} \mathrm{PB}(\mathrm{pH} 7.4)$, followed by addition of $0.5 \mathrm{~mL}$ liposomes $(2 \mathrm{mg} / \mathrm{mL})$ in $1 \mathrm{mM}$ PB (pH 7.4). To prepare LB-MSN-DT loaded with Alexa488 or IRDye $800 \mathrm{CW}$ labeled DT, plain DT was replaced with fluorescently labeled DT according the need of experiments. The mixture was incubated in an Eppendorf thermomixer (Nijmegen, The Netherlands) for $1.5 \mathrm{~h}$ at $25^{\circ} \mathrm{C}$ with a speed of $300 \mathrm{rpm}$. To remove the excess DT and liposomes, the suspension was centrifuged by using a Sigma 1-15 centrifuge (Osterode, Germany) for $5 \mathrm{~min}$ with a speed of 10,000 g. The resultant pellet was washed and re-dispersed in $1 \mathrm{mM} \mathrm{PB}$ (pH 7.4) for further use.

\section{Measurement of Size and Zeta Potential of LB-MSN-DT}

The size and zeta potential of LB-MSN-DT were determined by using dynamic light scattering (DLS) and laser Doppler velocimetry, respectively, with a Nano ZS® zetasizer (Malvern Instruments, Worcestershire, U.K.). The samples were diluted in $1 \mathrm{mM} \mathrm{PB}(\mathrm{pH} \mathrm{7.4)}$ to a concentration of $25 \mu \mathrm{g} / \mathrm{mL}$ (expressed based on the concentration of MSNs) and measured 3 times with 10 runs for each measurement.

\section{Determination of Encapsulation Efficiency (EE) and Loading Capacity (LC) of DT in LB-MSN-DT}

The loading efficiency of DT was determined by measuring the intrinsic fluorescence intensity of DT $\left(\lambda_{\text {ex }} 280 \mathrm{~nm} / \lambda_{\text {em }}\right.$ $320 \mathrm{~nm}$ ) in the supernatant before and after encapsulation by using a Tecan M1000 plate reader (Männedorf, Switzerland). The EE and LC were calculated using the equations below:

$$
\begin{aligned}
& \mathrm{EE}=\frac{M_{\text {loaded } D T}}{M_{\text {total } D T}} \times 100 \% \\
& \mathrm{LC}=\frac{M_{\text {loaded } D T}}{M_{M S N S}} \times 100 \%
\end{aligned}
$$

Where $M_{\text {loaded DT }}$ represents the mass of encapsulated DT, $M_{\text {total DT }}$ is the total amount of DT added to the formulation and $M_{M S N S}$ is the weight of MSNs.

\section{In Vitro Release of DT from LB-MSN-DT}

To study the release of DT, $1 \mathrm{~mL}$ nanoparticle suspension with a concentration of $1 \mathrm{mg} / \mathrm{mL}$ (expressed based on the concentration of MSNs, corresponding to about $0.2 \mathrm{mg} / \mathrm{mL}$ DT) in PBS was incubated for one month at $37^{\circ} \mathrm{C}$ by using an Eppendorf thermomixer (Nijmegen, The Netherlands) set at a speed of $550 \mathrm{rpm}$. At predetermined time points, the samples were centrifuged for $5 \mathrm{~min}$ with a speed of 10,000 g. $600 \mu \mathrm{L}$ sample from the supernatant was collected and the amount of DT was measured by intrinsic fluorescence intensity of DT. Fresh PBS with the same volume of the collected supernatant was added back to the suspension. The release percentage of DT was calculated by dividing the released amount of DT by the total amount of DT initially loaded in LB-MSN-DT.

\section{Modification of Microneedle Arrays to Achieve a PH-Sensitive Surface}

Silicon microneedle arrays with 576 microneedles per array on a back plate of $5 \times 5 \mathrm{~mm}^{2}$ with a microneedle length of $200 \mu \mathrm{m}$ were kindly provided by Robert Bosch $\mathrm{GmbH}$ (Stuttgart, Germany). To obtain pH-sensitive microneedles, the surface was modified with pyridine groups as previously reported (20). In brief, the microneedle surface was first cleaned by piranha solution $(70 \%$ sulfuric acid and 30\% hydrogen peroxide) at $120^{\circ} \mathrm{C}$ for $2 \mathrm{~h}$. Caution: piranha is strongly acidic and oxidizing. Piranha reacts violently with organic compounds, and it should not be stored in closed containers. Subsequently, the microneedles were extensively washed with MilliQ water followed by washing with acetone and methanol. Next, the microneedles were incubated in 2\% APTES in toluene overnight to obtain an amine-modified surface and thereafter incubated with 4-pyridinecarboxaldehyde (100 mM) in anhydrous isopropanol containing $1 \%$ acetic acid overnight. Finally, the formed imine bonds were reduced to secondary amines by incubating the microneedles with $\mathrm{NaBH}_{3} \mathrm{CN}$ $(50 \mathrm{mM})$ in isopropanol for $2 \mathrm{~h}$. After cleaning the microneedles were stored under vacuum at $50^{\circ} \mathrm{C}$ until further use.

\section{Multilayer Coating of LB-MSN-DT on the Surface of Microneedle Arrays}

LB-MSN-DT and TMC were alternately coated onto the surface of microneedle arrays by using a layer-by-layer approach. The $\mathrm{pH}$-sensitive microneedle arrays were transferred into Greiner Cellstar ${ }^{\circledR} 48$ well plates. $50 \mu \mathrm{L}$ negatively charged LB-MSN-DT $(0.5 \mathrm{mg} / \mathrm{mL})$ in $1 \mathrm{mM} \mathrm{PB}(\mathrm{pH} 5.8)$ was added onto the top of each microneedle array and the arrays were incubated for $30 \mathrm{~min}$. The excess nanoparticles were washed by adding $450 \mu \mathrm{L} 1 \mathrm{mM}$ PB (pH 5.8). Next, the microneedle arrays were dried under pressurized nitrogen 
flow for $10 \mathrm{~min}$. After the first coating layer of LB-MSN-DT, $50 \mu \mathrm{L}$ positively charged TMC $(40 \mu \mathrm{g} / \mathrm{mL})$ in $1 \mathrm{mM} \mathrm{PB}$ (pH 5.8) was added onto the top of each microneedle array and the arrays were incubated with TMC for another $30 \mathrm{~min}$. The concentration of LB-MSN-DT and TMC in the coating solutions were chosen based on prior studies $(11,23)$. The excess TMC was removed by washing the microneedle arrays with $450 \mu \mathrm{L} 1 \mathrm{mM}$ PB (pH 5.8). Subsequently, the microneedle arrays were dried under nitrogen flow as described above. This procedure was repeated until the desired number of coating layers of LB-MSN-DT was reached. After the last layer of LB-MSN-DT, no more TMC was coated onto the microneedle surface. In order to study the dose effect of DT using coated microneedles, the microneedle arrays were coated with either 5 or 3 layers of LB-MSN-DT and 4 or 2 alternate layers of TMC, respectively.

To determine the coating efficiency of DT on microneedles, the amount of nano-encapsulated DT in the supernatant after washing was determined by measuring the intrinsic fluorescence of DT. The coating efficiency was calculated by dividing the amount of coated DT by the total amount of DT initially added to the coating solution.

\section{Insertion of Microneedle Arrays into Ex Vivo Human Skin}

Ex vivo human skin was obtained from a local hospital according to Helsinki principles. A written informed patient consent was obtained. To reproducibly insert the microneedles into the skin, an in-house developed impact-insertion injector together with a uPRAX applicator controller (Delft, The Netherlands) was used by using either a single insertion mode or multiple insertion mode $(27,28)$. In case of a single insertion, the microneedle arrays were inserted into the skin with an average velocity of $0.5 \mathrm{~m} / \mathrm{s}$ and kept in the skin for $30 \mathrm{~min}$ by applying a force of $5 \mathrm{~N}$ on top of the microneedle array. In case of multiple insertion mode, the microneedle arrays were 10 times inserted into the skin within $10 \mathrm{~s}$ with an average velocity of $0.5 \mathrm{~m} / \mathrm{s}$. After the last penetration, the microneedles were removed from the skin.

\section{Visualization of the Coated Microneedles before and after Penetration of $E x$ Vivo Human Skin by Scanning Electronic Microscopy (SEM)}

The 5-layer LB-MSN-DT coated microneedles were visualized with a Nova NanoSEM (Eindhoven, The Netherlands) operated with a voltage of $15 \mathrm{kV}$ before and after removal from the skin. To increase the surface conductivity, the microneedle arrays were coated with a layer of platina/ palladium before visualization.

\section{Release of LB-MSN-DT from Microneedle Arrays into Ex Vivo Human Skin}

To visualize the release of LB-MSN-DT from microneedle arrays into the skin, the 5-layer LB-MSN-DT coated microneedle arrays and the released nanoparticles in the skin were visualized by using a Nikon D-Eclipse C1 CLSM (Tokyo, Japan). For this purpose, DT-Alexa488 and TMCRho were used. The coated microneedles and the skin area penetrated by coated microneedles were scanned with a depth resolution of $5 \mu \mathrm{m} /$ step by using a $10 \times$ and $4 \times$ Plan Apo objective, respectively. An argon laser (488 nm) with a 530/ 55 emission filter and a diode-pumped solid-state laser (561 nm) with a 590/55 emission filter were used for visualization of DT-Alexa488 and TMC-Rho, respectively.

The released amount of DT in the ex vivo human skin was quantified by using a Perkin-Elmer IVIS Lumina Series III in vivo imaging system (Waltham, MA, USA). For this purpose, DT was labeled with IRDye $800 \mathrm{CW}$ (DT-IRDye800) by using a IRDye 800CW protein labeling kit (low molecular weight) according to the manufacturer's instruction. The LB-MSN-DT-IRDye800 coated microneedles were inserted into human skin by using either the single or multiple insertion mode as described above. A calibration curve was prepared by injecting different amounts of LB-MSN-DT-IRDye800 in the skin by using a hollow microneedle (see below). To determine the amount of DT released from the coated microneedles, the fluorescence intensity of DT-IRDye800 in the skin was measured by using the in vivo imaging system with a $745 \mathrm{~nm}$ excitation wavelength and an ICG emission filter. By using the calibration curve the amount of delivered DT was calculated.

\section{Hollow Microneedles and Applicator}

The hollow microneedles were prepared by etching of fused silica capillaries with hydrofluoric acid, as previously described (29). In brief, silica capillaries (375 $\mu \mathrm{m}$ outer diameter, $50 \mu \mathrm{m}$ inner diameter) were cut into $4-\mathrm{cm}$ pieces and filled with silicone oil in a vacuum oven $\left(100^{\circ} \mathrm{C}\right)$ overnight. The tips of capillaries were etched in $\geq 48 \%$ hydrofluoric acid for $4 \mathrm{~h}$. Subsequently, the polyimide coating was removed by dipping the microneedle tips into hot sulfuric acid $\left(250^{\circ} \mathrm{C}\right)$ for $5 \mathrm{~min}$. The applicator for hollow microneedles consists of a syringe pump and an injector for precise control of injection depth, rate and volume. The hollow microneedles, injector and pump were connected by silica capillaries and high-pressure resistant CapTite ${ }^{\mathrm{TM}}$ connectors (24).

\section{Immunization Studies in Mice}

Female BALB/c mice of 7-8 weeks old (Charles River, Maastricht, The Netherlands) at the start of the experiments 
were used for the immunization study. The animals were housed under standardized conditions in the animal facility of Leiden Academic Centre for Drug Research. The study was approved by the ethical committee on animal experiments of Leiden University (Licence number 14166).

Mice were first anesthetized by intraperitoneal injection of ketamine $(60 \mathrm{mg} / \mathrm{kg}$ ) and xylanize $(4 \mathrm{mg} / \mathrm{kg}$ ) before shaving the abdomen area. In case of coated microneedles, the LBMSN-DT coated microneedle arrays were inserted into the abdomen of mice by using the multiple insertion mode as described above for the studies in ex vivo human skin. Each mouse was immunized with one microneedle array coated with either 5 or 3 layers of LB-MSN-DT. In case of hollow microneedles, the following groups were included: a) $10 \mu \mathrm{L}$ suspension of LB-MSN-DT, b) $10 \mu \mathrm{l}$ DT solution and c) $10 \mu \mathrm{l}$ DT and TMG solution. All formulations of hollow microneedle groups contained $0.31 \mu \mathrm{g}$ DT. The same amount of TMC was included in the DT and TMC group. The formulation was injected into the skin of the abdomen of mice with a rate of $10 \mu \mathrm{L} / \mathrm{min}$ at a depth of $120 \mu \mathrm{m}$. Subcutaneously injected $5 \mu \mathrm{g}$ DT formulated with $150 \mu \mathrm{g}$ colloidal aluminum phosphate (DT-Alum) in PBS with a volume of $100 \mu \mathrm{L}$ was used as a positive control. The mice were immunized on day 0 (prime), 21 (1 st boost), 42 (2nd boost) and sacrificed on day 56 . The serum was withdrawn from the tail veins of the mice on day 0,21 and 42 prior to the immunization. On day 56 the serum was collected from femoral vein and the mice were sacrificed by cervical dislocation.

\section{Measurement of DT-Specific Antibody Titers}

The total IgG and subtype IgG1 and IgG2a titers in the serum were measured by using ELISA as previously reported (30). Briefly, the wells of 96-well plates were first coated with $140 \mathrm{ng}$ DT overnight at $4^{\circ} \mathrm{C}$. Next, the plates were blocked with $1 \%$ BSA and appropriate 3-fold serial diluted serum samples were applied to the plates and incubated for $2 \mathrm{~h}$ at $37^{\circ} \mathrm{C}$. Subsequently, HRP-conjugated goat anti-mouse total $\operatorname{IgG}$, IgG1 and IgG2a were added into the wells and incubated for $1.5 \mathrm{~h}$. Finally, TMB was added to the plates and $2 \mathrm{M}$ sulfuric acid was added to stop the reaction. The absorbance was measured at $450 \mathrm{~nm}$ by using a Tecan M1000 plate reader. The antibody titers were expressed as the ${ }^{10} \log$ value where the corresponding absorbance is located in the middle of the S-shaped dilution-absorbance curve.

\section{Measurement of DT-Neutralizing Antibody Titers}

To check the functionality of the antibodies, diphtheria toxin neutralizing antibody titers in the serum of the mice at day 56 were checked by using a Vero-cell assay (31). Briefly, appropriate 2-fold serial diluted serum was first applied to 96-well plates. $5 \times 10^{-5}$ Lf diphtheria toxin was added to each well and incubated for $2 \mathrm{~h}$ at $37^{\circ} \mathrm{C}$ in a stove with $5 \% \mathrm{CO}_{2}$. Subsequently, $1.25 \times 10^{4}$ Vero cells were added to each well and incubated for 6 days at $37^{\circ} \mathrm{C}$ in the stove with $5 \% \mathrm{CO}_{2}$. Finally, the neutralizing antibodies were shown as the ${ }^{2} \log$ value of the highest dilution times of serum that protected the Vero cells.

\section{Statistics Analysis}

All the data of antibody titers were analyzed by one way ANOVA with Newman-Keuls Multiple post-test by using GraphPad Prism software (version 5.02). The level of significance was set at $* p<0.05, * * p<0.01, * * * p<0.001$.

\section{RESULTS}

\section{Physicochemical Characteristics of LB-MSN-DT}

The physicochemical characteristics of LB-MSN-DT are shown in Table I. The size of LB-MSN-DT was approximately $700 \mathrm{~nm}$ with a polydispersity index (PDI) slightly larger than 0.3. The nanoparticles showed a high negative zeta potential. DT was efficiently encapsulated into the nanoparticles with a high EE and LC.

\section{In Vitro Release of DT from LB-MSN-DT}

The in vitro release of DT was investigated by suspending LBMSN-DT in PBS for one month. As shown in Fig. 1, there was a moderate burst release of DT of about $20 \%$ within the first day, followed by a sustained release, reaching a total release percentage of about $70 \%$ on day 30 . These results indicate that the LB-MSN-DT may serve as a reservoir and allow the sustained release of DT, but at the same time retain sufficient DT for a prolonged period of time to deliver it as nanoparticulate antigen to APCs.

\section{Quantification of Coated Amount of Nano-Encapsulated DT on Microneedle Arrays}

As shown in Fig. 2a, the amount of nano-encapsulated DT that was coated onto the microneedles increased linearly with increasing number of coating layers. About $0.4 \mu \mathrm{g}$ DT was coated onto the microneedles of one microneedle array per layer. The coating efficiency was similar for each layer and was about 20-26\% (Fig. 2b). As shown in Table II, the cumulative amount of nano-encapsulated DT coated on the microneedle surfaces of one microneedle array was about $1.9 \mu \mathrm{g}$ and $1.1 \mu \mathrm{g}$, corresponding to $9.7 \mu \mathrm{g}$ and $5.7 \mu \mathrm{g} \mathrm{LB}-$ MSN-DT (based on the mass of MSNs) for a 5-layer and 3layer coating, respectively. 
Table I Physicochemical Characteristics of LB-MSN-DT $(n=3)$.

\begin{tabular}{llllll}
\hline Nanoparticles & Size $^{\mathrm{a}}(\mathrm{nm})$ & PDI $^{\mathrm{b}}$ & $\mathrm{ZP}^{\mathrm{C}}(\mathrm{mV})$ & EE\% $^{\mathrm{d}}$ & LC\% $^{\mathrm{e}}$ \\
\hline LB-MSN-DT & $676 \pm 7$ & $0.322 \pm 0.016$ & $-35 \pm 1$ & $77.1 \pm 6.4$ & $19.3 \pm 1.6$ \\
\hline
\end{tabular}

Data are average \pm SEM of 3 independent batches

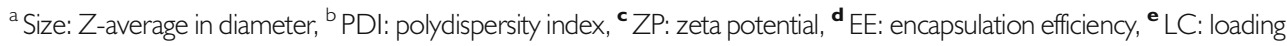
capacity

\section{Visualization of Coated Microneedles before and after Penetrating $E x V i v o$ Human Skin by SEM}

The 5-layer LB-MSN-DT coated microneedles were visualized by SEM. The uncoated $\mathrm{pH}$-sensitive microneedles showed a smooth surface (Fig. 3a, b I-b2). On the surface of LB-MSN-DT coated microneedles (Fig. 3 c l-c2), single nanoparticles or clusters of nanoparticles were observed. After insertion of the microneedles into and removal from the skin, the nanoparticle density was reduced on the microneedle surface (Fig. $3 \mathrm{~d} \mathbf{I}-\mathbf{d} \mathbf{2}$ ).

\section{Visualization of the Released LB-MSN-DT in Ex Vivo Human Skin}

After observation of the reduction of the number of nanoparticles on the microneedle surface after penetration in and withdrawal from human skin, CLSM was used to visualize the released LB-MSN-DT in the skin. To this end, the 5layer LB-MSN-DT coated microneedles before penetration of the skin were first visualized. The green color from DTAlexa488 (Fig. 4a) and red color from TMC-Rho (Fig. 4b) were observed and they colocalized on the surface of the microneedles (Fig. 4c). These results support the SEM images of LB-MSN-DT coated microneedles (Fig. 3 c I-c2), further revealing that LB-MSN-DT were successfully coated onto the surface of the microneedles.

Next, the released LB-MSN-DT in the skin was visualized by CLSM. After a single insertion, the fluorescence of the

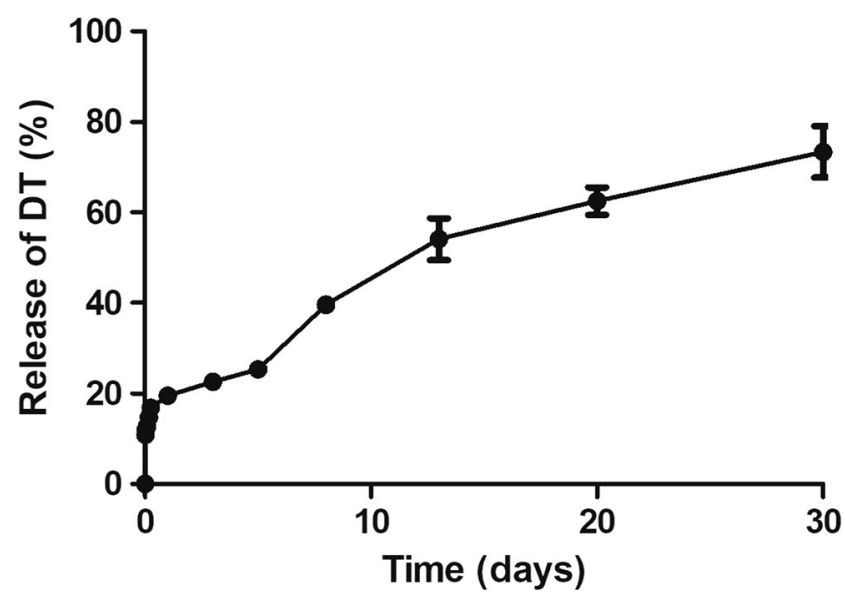

Fig. I In vitro release of DT from LB-MSN-DT in PBS at $37^{\circ} \mathrm{C}$ as a function of time. Bars represent mean $\pm \mathrm{SEM}, n=3$. released DT-Alexa488 and TMC-Rho were clearly observed (Fig. 4d-f). The green color from DT-Alexa488 (Fig. 4d) and red color from TMC-Rho (Fig. 4e) co-localized in the microchannels induced by the microneedles (Fig. 4f). After the microneedles were inserted in and withdrawn from the skin by using the multiple insertion mode, clearly more microchannels were observed as indicated by the fluorescence of DT-Alexa488 and TMC-Rho (Fig. 4g-i). These results together with the SEM images of the coated microneedles after penetration of the skin indicate that LB-MSN-DT were successfully released into skin.

\section{Quantification of the Released Amount of DT from Microneedles into Ex Vivo Human Skin}

As shown in Table II, after insertion of the 5-layer coated microneedle arrays into ex vivo human skin, the delivery efficiency from the microneedles by using the multiple insertion mode $(42.8 \%)$ was more than twice as high compared to that in single insertion mode $(17.9 \%)$. Based on this observation, the multiple insertion mode was chosen for subsequent penetration studies. Next, the released amounts of DT from microneedles coated with 5 and 3 layers of LB-MSN-DT were compared. The amount of delivered DT in the skin from one 5-layer coated microneedle array $(0.814 \mu \mathrm{g})$ was about 3-fold higher than that from a 3-layer coated microneedle array $(0.256 \mu \mathrm{g})($ Table II).

\section{IgG Antibody Titers after Intradermal Vaccination}

Total IgG titers are shown in Fig. 5. On day 21 all groups showed detectable total IgG titers (Fig. 5a). On day 42, the responses of all groups increased compared to those on day 21. Responses of hollow microneedle injected LB-MSN-DT were significantly higher than those induced by DT/TMC solution and LB-MSN-DT coated microneedle groups (Fig. 5b) (p < 0.05). On day 21 and 42, DT-Alum induced higher total IgG responses than other groups, probably due to the much higher dose used $(p<0.01)$. On day 56 , the responses of hollow microneedle injected LB-MSN-DT and 5-layer LBMSN-DT coated microneedles increased to similar IgG levels as those induced by DT-Alum, despite the ca. 15-fold lower dose, while DT/TMC solution elicited significantly lower levels than DT-Alum. 
Fig. 2 Cumulative amount of nano-encapsulated DT (a) that was coated on the microneedles of one microneedle array and coating efficiency (b) as a function of the number of layers. Data is represented as average \pm SEM of 3 independent microneedle arrays.
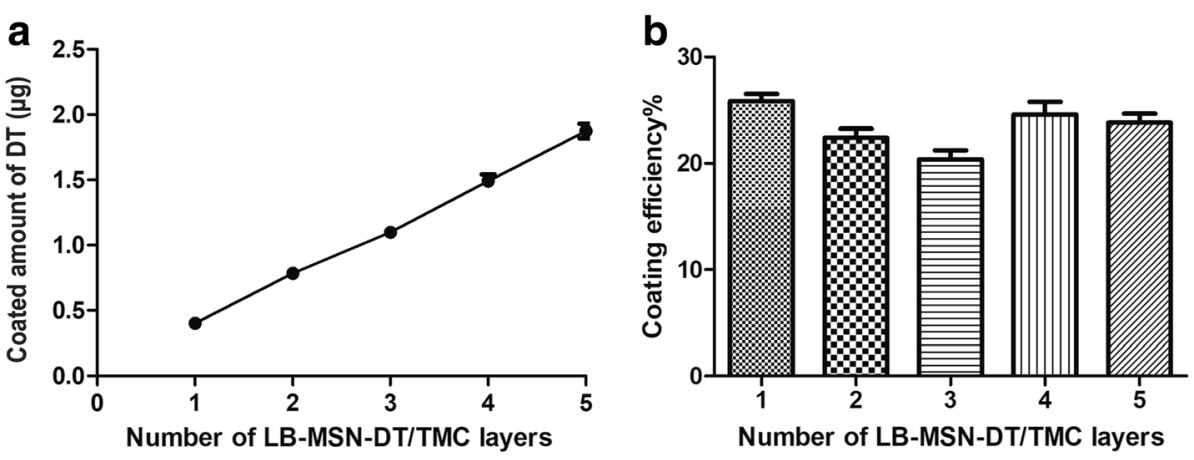

In all these three immunizations, the addition of TMC did not improve the total IgG response. Additionally, 5-layer LBMSN-DT coated microneedles seemed to induce a stronger total $\mathrm{IgG}$ response than 3-layer coated microneedles, although the difference was not significant ( $\mathrm{p}$ 70.05). In summary, LBMSN-DT delivered by both coated and hollow microneedles successfully induced DT-specific total IgG responses. LBMSN-DT induced superior total IgG responses as compared to DT/TMG solution when administered by hollow microneedles (after 1st boost), but not when using coated microneedles.

Besides total $\mathrm{IgG}$, we measured the subtype $\mathrm{IgG} 1$ and IgG2a titers. As shown in Fig. 6, IgG1 followed the trend of total IgG (Fig. 6a, c, e). Hollow microneedle injected LBMSN-DT induced stronger responses than DT/TMC solution (after 1st boost). However, this advantage of using LBMSN-DT disappeared when LB-MSN-DT were delivered by coated microneedles. In case of IgG2a titers, on day 21 all groups except coated microneedles induced detectable IgG2a titers (Fig. 6b). On day 42, DT-Alum induced significantly higher titers than other groups (Fig. 6d) (p;0.05). Although not significant, hollow microneedle injected LBMSN-DT seemed to induce a higher IgG2a response compared to DT solution $(p=0.10)$ and coated microneedles $(p=$ 0.12). On day 56, hollow microneedle injected LB-MSN-DT and DT solution showed significantly higher IgG2a titers than 3-layer LB-MSN-DT coated microneedle group ( $\mathrm{p} 70.01$ ), but this was not significant compared to 5-layer LB-MSN-DT coated microneedles (Fig. 6f) $(p=0.15)$. Furthermore, the IgG2a titers induced by hollow microneedle injected LBMSN-DT reached a level similar to those induced by DTAlum. In summary, hollow microneedle injected LB-MSNDT induced stronger IgG1 and IgG2a titers than LB-MSNDT coated microneedles.

The functionality of the antibody response was determined by measuring the DT-neutralizing antibodies from serum taken on day 56. As expected, the subcutaneously injected DTAlum with a high dose induced high neutralizing antibody titers (Fig. 7). Hollow microneedle-injected LB-MSN-DT showed a significant higher neutralizing response than a mixture of DT and TMC solution and coated microneedle groups.

\section{DISCUSSION}

Microneedle technologies for the intradermal delivery of drugs, including vaccines, have been extensively investigated during the past twenty years (32). As the skin contains a large number of APCs, such as epidermal Langerhans cells and dermal dendritic cells, microneedles have gained particular attention as attractive delivery systems for intradermal vaccination (33). In this study, we investigated the immunogenicity of DT encapsulated MSNs after coated microneedle- and hollow microneedle-mediated intradermal immunization in mice. We showed that LB-MSN-DT delivered by both coated

Table II Coated and Released Amount of DT/LB-MSN-DT from the Microneedles of a Single Microneedle Array $(n=3)$.

\begin{tabular}{|c|c|c|c|c|c|c|}
\hline \multirow[t]{2}{*}{ Microneedles } & \multirow[t]{2}{*}{ Coated DT $(\mu g)$} & \multirow[t]{2}{*}{${ }^{a}$ Coated LB-MSN-DT $(\mu g)$} & \multicolumn{2}{|l|}{${ }^{\mathrm{b}}$ Delivered DT $(\mu \mathrm{g})$} & \multicolumn{2}{|l|}{ `Delivered percentage (\%) } \\
\hline & & & Multiple insertion mode & Single insertion mode & Multiple insertion mode & Single insertion mode \\
\hline 5-layer coated & $1.9 \pm 0.1$ & $9.7 \pm 0.2$ & $0.814 \pm 0.008$ & $0.341 \pm 0.083$ & $42.8 \pm 0.1 \%$ & $17.9 \pm 0.8 \%$ \\
\hline 3-layer coated & $1.1 \pm 0.1$ & $5.7 \pm 0.2$ & $0.256 \pm 0.001$ & - & $23.2 \pm 0.0 \%$ & - \\
\hline
\end{tabular}

Data are average \pm SEM of 3 independent microneedle arrays

a The coated amount of LB-MSN-DT is expressed as the mass of MSNs and was calculated by using the coated amount of DTand loading capacity of DT in LBMSN-DT

b The delivered dose of DTwas measured in ex vivo human skin

' Delivered percentage was calculated by dividing the delivered amount of DT in ex vivo human skin by the coated amount of DT on the microneedles 

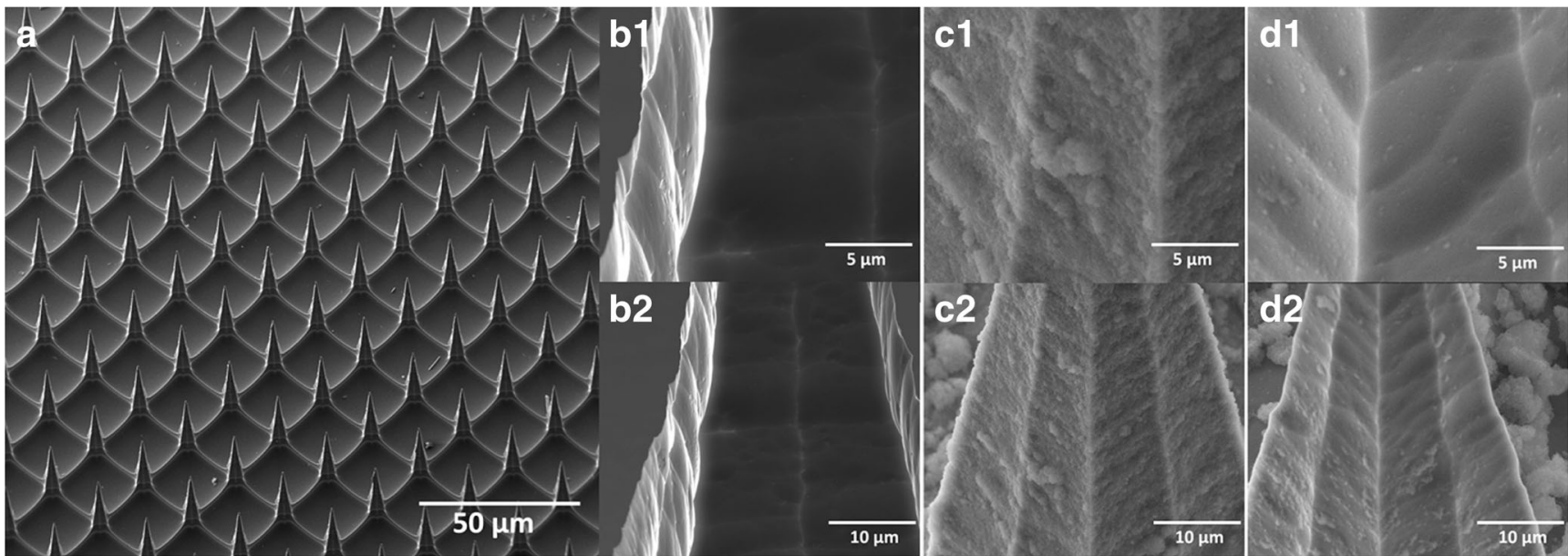

Fig. 3 Scanning electron microscopy (SEM) images of uncoated pH-sensitive microneedles ( $a$, b l-b2), microneedles coated with 5 layers of LB-MSN-DT/TMC $(\mathrm{cl}-\mathrm{c} 2)$, and the microneedles after insertion into and removal (multiple insertion mode) from ex vivo human skin ( $\mathrm{d} \mathrm{l}-\mathrm{d} 2$ ).

and hollow microneedles induced DT-specific antibody titers. Both the nano-encapsulation of DT and the type of microneedles were found to affect the immune responses.

Nanoparticulate vaccines have been reported to enhance the immunogenicity of antigens by increasing their uptake by APCs (3,34). In this study, MSNs were chosen for the loading of DT as they have large pores which allow for efficient loading of antigen (11). In a previous study it was shown that ovalbumin (OVA) loaded MSNs were able to elicit antibody responses with a reduced antigen dose compared to OVA solution adjuvanted with QuilA (9). In another study, MSNs loaded with a virus related antigen induced 10-fold higher antibody responses than the mixture of the antigen and an immune modulator (10). Our findings are in line with these results, as we showed that hollow microneedle injected LBMSN-DT induced distinctly higher total IgG and IgG1 titers as compared to a solution of plain DT.

When coating LB-MSN-DT onto the microneedle arrays, the coated amount of DT per layer on one microneedle array (about $500 \mathrm{ng}$ ) was higher than that reported in a previous study (about $300 \mathrm{ng}$ ) where plain DT was coated onto the same type of microneedle arrays (23). The high loading

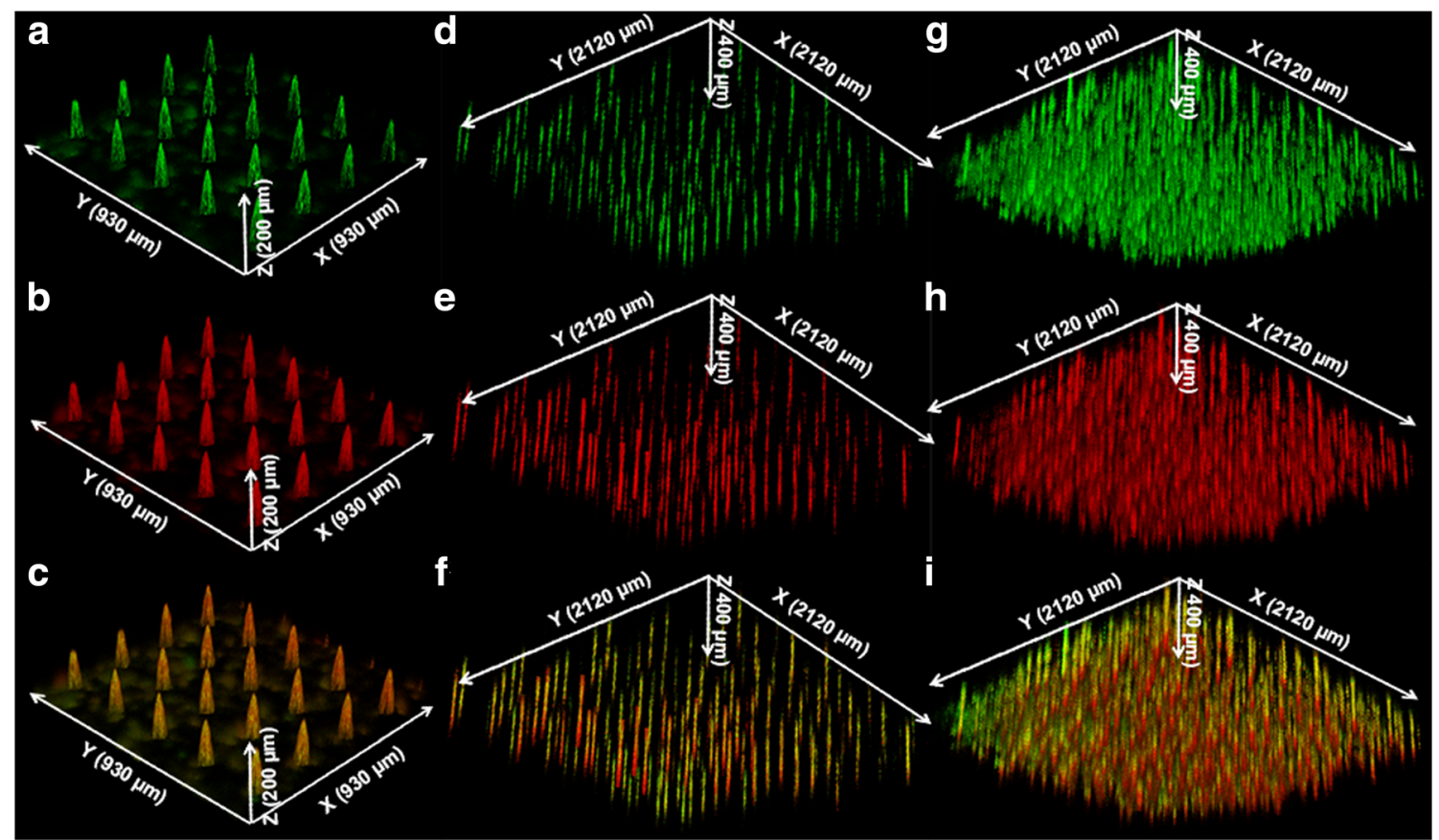

Fig. 4 Confocal laser scanning microscopy (CLSM) images of 5-layer LB-MSN-DT coated microneedles (a: DT-Alexa488; b: TMC-Rho; c: merged), and ex vivo human skin after insertion and removal of microneedle arrays (5-layer coated) by using single (d: DT-Alexa488; e: TMC-Rho; f: merged) or multiple insertion mode (g: DT-Alexa488; $\mathbf{h}$ : TMC-Rho; i: merged). 
D21

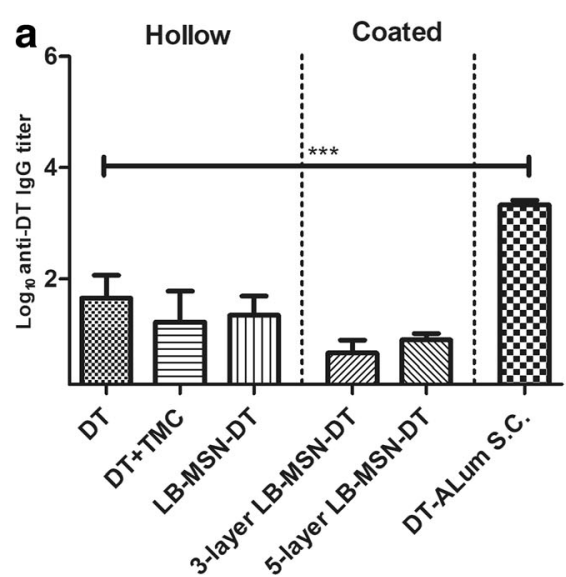

D42

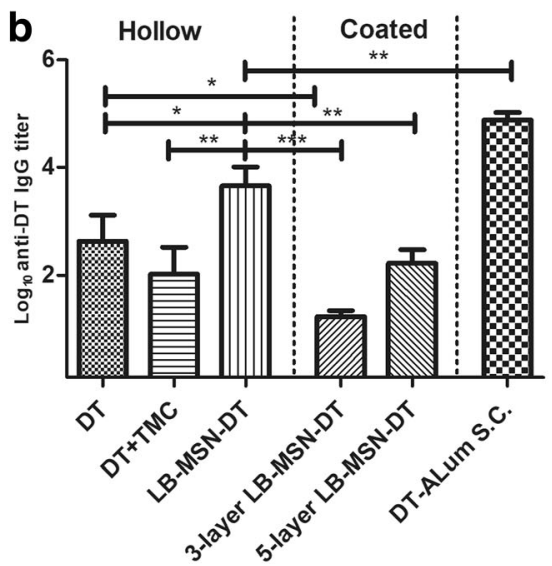

D56

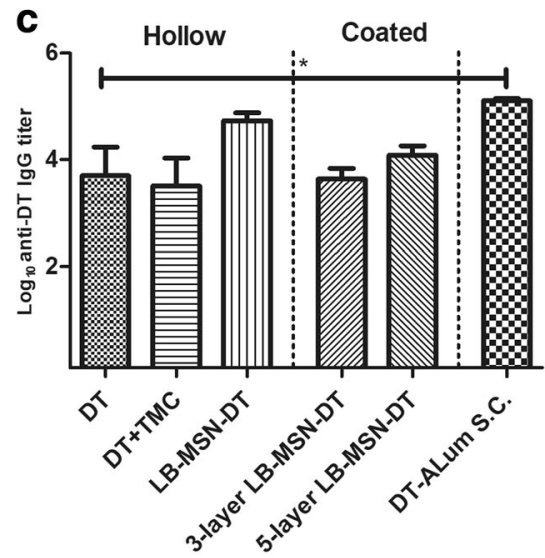

Fig. 5 DT-specific total lgG antibody titers on day 21 (a), 42 (b) and 56 (c). Bars represent mean \pm SEM, $n=8$. $* p<0.05$, *** $p<0.01$, **** $p<0.001$.

Fig. 6 DT-specific $\lg G \mid(\mathbf{a}, \mathbf{c}, \mathbf{e})$ and $\lg \mathrm{g} 2 \mathrm{a}(\mathbf{b}, \mathbf{d}, \mathbf{f})$ antibody titers on day 2 I (a, b), 42 (c, d) and 56 $(\mathbf{e}, \mathbf{f})$. Bars represent mean \pm SEM $n=8 . * p<0.05, * * * 10.01$, **** $p<0.001$. $\lg G 1$
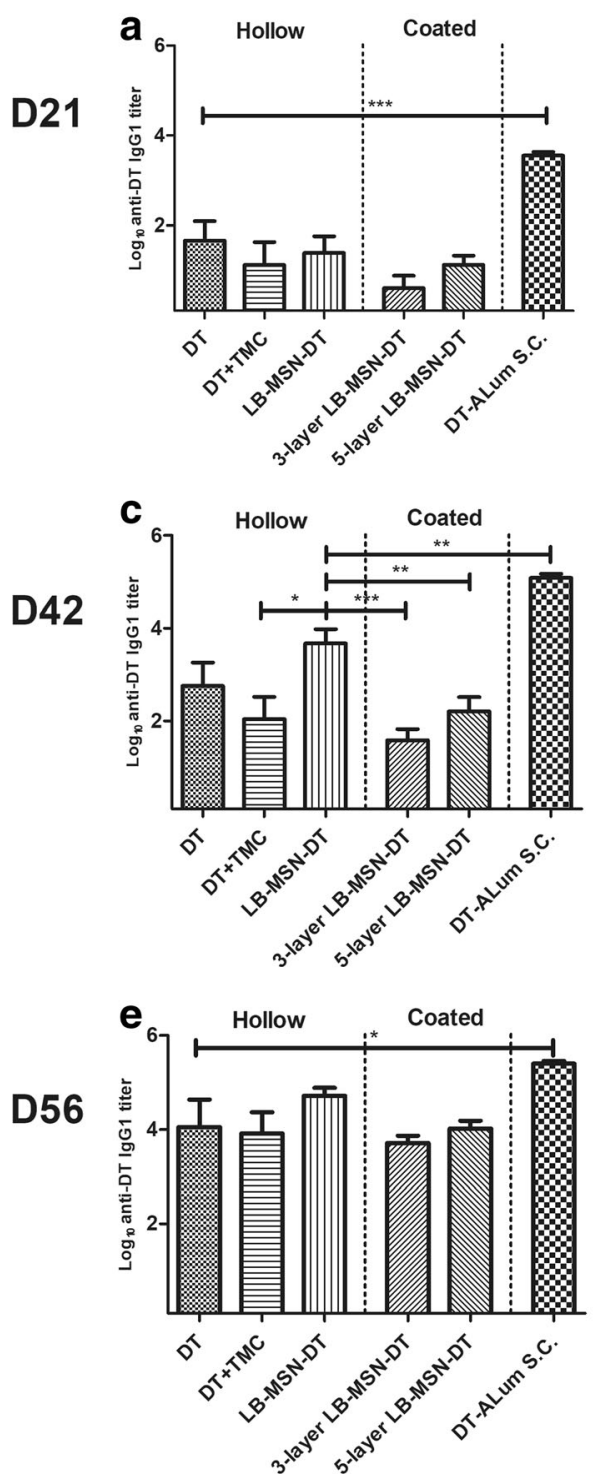

$\lg$ Ga
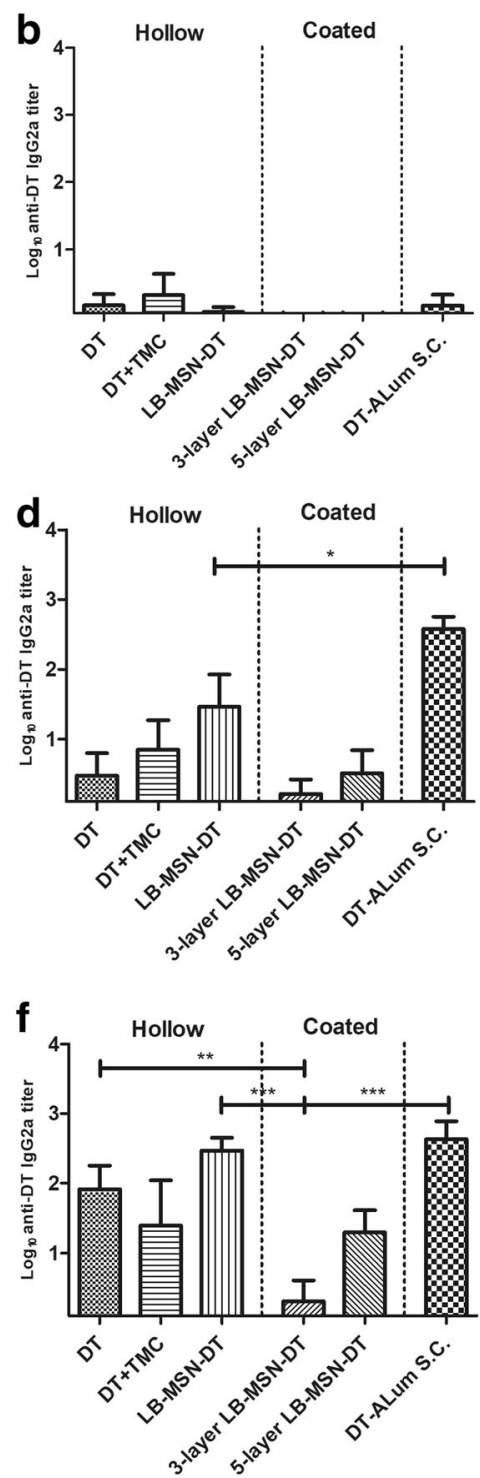


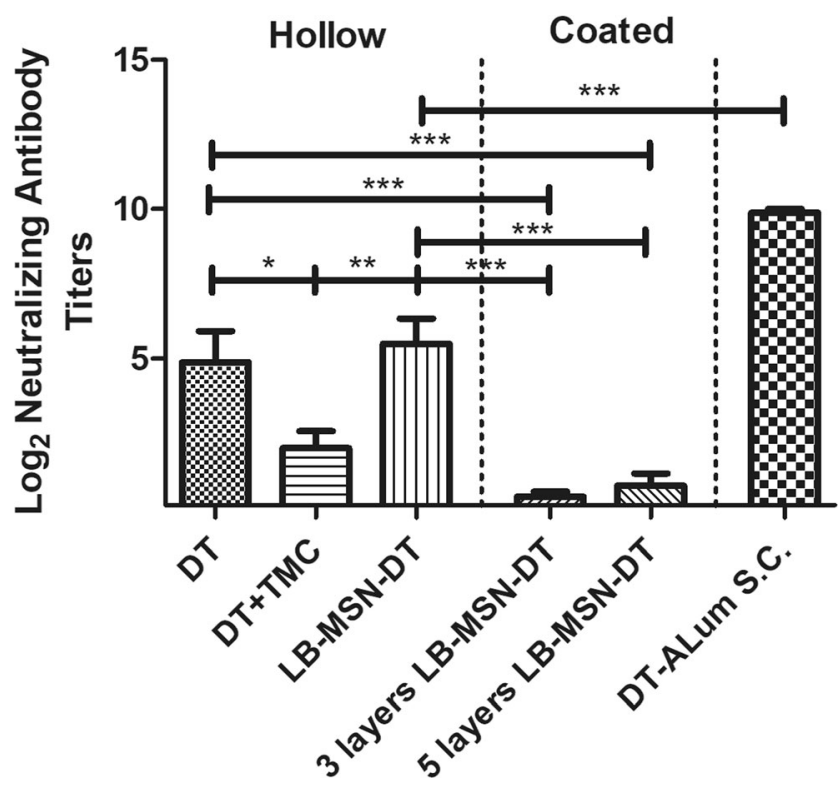

Fig. 7 DT-neutralizing antibody titers of mice. Results are shown for serum collected on day 56 . Bars represent mean \pm SEM,$n=8$. ${ }_{p} p<0.05$, **** $p<0.001$.

capacity of DT in LB-MSN-DT together with the high surface charge of LB-MSN-DT may synergistically lead to this higher coating amount. Additionally, the multilayer coating approach used in the current study can further increase the coated amount of antigen by increasing the number of coating layers. By adjusting the number of coating layers, the coated amount of nanoparticles/antigen can be tailored.

Besides the successful coating of antigen on microneedles, it is important to have a fast release of the coating after the microneedles were penetrated into skin. Here we showed that by using a multiple insertion mode (10 penetrations within $10 \mathrm{~s}$ ), the released amount of antigen was increased by 2.5fold as compared to a single insertion mode. The amount of DT released into the skin was also increased compared to that released from the 5 layer coatings of plain DT using a single penetration (23). Therefore, the combination of multiple insertions with nanoparticle coatings may require less coating layers, which will facilitate the production process of coated microneedles. When using multiple insertions, the application time was much shorter than that used in single penetration mode. The improvement of release efficiency may be due to the friction force between the microneedles and the skin tissue when the microneedles were inserted in and removed from the skin. The short wearing time of microneedles by using the multiple insertion mode might help improving the acceptance by vaccinees. Nevertheless, the drawback of using the multiple insertion mode is that a sophisticated applicator needs to be developed. In the multilayer coating approach described in the current study, the TMC used has strong adhesion properties and could prevent the coated nanoparticles releasing from the microneedles (35). It would therefore be interesting to examine polymers which have weaker electrostatic interactions with the microneedles.

While hollow microneedle injected LB-MSN-DT induced a stronger immune response as compared to plain DT, LBMSN-DT delivered by coated microneedles induced a comparable response as DT/TMC solution. The results of coated microneedles are in contrast with those reported in a recent study, which showed that nanoparticulate vaccine coated microneedles induced superior immune responses as compared to antigen solution intradermally delivered by a hypodermic needle (36). One explanation is that the dose delivered by coated microneedles may be lower than that delivered by hollow microneedles in mice skin in vivo. However, there are at least two arguments against this hypothesis. Firstly, our results showed that the coated microneedles delivered a two-fold higher (5-layer coated) or comparable (3-layer coated) dose in ex vivo human skin, respectively, compared to that delivered by hollow microneedles in human skin. The stratum corneum, viable epidermis and dermis of mouse skin are much thinner than that of human skin (37). However, the trigger to release the coating from the microneedles is the environmental $\mathrm{pH}$. In the epidermis and dermis in mouse and human skin, the $\mathrm{pH}$ is 7.4. Therefore, the difference of the skin thickness between mouse and human skin is not expected to change the delivery efficiency. Secondly, a previous study showed that the delivered amount of DT from 5-layer coated microneedles into ex vivo human skin was similar as that delivered in ex vivo mouse skin (23). To summarize, the lower than expected responses of coated microneedle delivered LB-MSN-DT was not likely caused by lower dose of DT delivered.

Although the LB-MSN-DT coated microneedles induced similar total IgG and IgG1 responses as compared to hollow microneedle injected LB-MSN-DT on day $56(p>0.05)$, they induced distinctly lower IgG2a responses. At the same time, it has been reported that nano-encapsulation of antigen can increase IgG2a responses $(24,38)$. These results suggest that the advantage of using nanoparticles is abrogated when they are delivered by coated microneedles. One possible explanation for the lower response induced by coated microneedles is that the nanoparticles were not released from the nanoparticle/TMC layers after their deposition in the skin. As a result, the nanoparticles may be not efficiently taken up by APGs or drained to lymph nodes. In the hollow microneedle groups, the addition of TMC did not improve the immune responses either. An adjuvant effect of TMC has been reported for hypodermic needle-mediated intradermal vaccination (39). This inconsistency may be caused by the much lower dose of TMC used in our study.

Previous studies have shown that IgG1 titers may be mainly responsible for the neutralizing titers against diphtheria toxin (31). However, our results showed that although hollow microneedle and coated microneedle groups induced IgG1 responses close to those induced by DT-Alum, they still 
induced much lower neutralizing antibodies. These results indicate that the IgG1 titers may need to reach a certain threshold in order to achieve protection against diphtheria toxin.

\section{CONCLUSION}

In this study, we showed that DT loaded MSNs can be successfully delivered into mice by using coated and hollow microneedles, and evoke DT specific antibody responses. When inserting coated microneedles into skin, the multiple insertion mode of the applicator significantly increased the release efficiency of the coating compared to the single insertion mode. DT encapsulated in MSNs induced a stronger antibody response than antigen solution when delivered by hollow microneedles (after 1st boost), but not by coated microneedles. Our results revealed that both the nanoencapsulation of DT and the type of microneedles affect the immunogenicity of the antigen.

\section{ACKNOWLEDGEMENTS AND DISCLOSURES}

We thank Hilde Vrieling and Amy Kogelman from Intravacc for their help with the neutralizing antibody assay. Guangsheng Du acknowledges the support from China Scholarship Council.

Open Access This article is distributed under the terms of the Creative Commons Attribution 4.0 International License (http://creativecommons.org/licenses/by/4.0/), which permits unrestricted use, distribution, and reproduction in any medium, provided you give appropriate credit to the original author(s) and the source, provide a link to the Creative Commons license, and indicate if changes were made.

\section{REFERENCES}

1. Greenwood B. The contribution of vaccination to global health: past, present and future. Philosophical transactions of the Royal Society of London Series B. Biol Sci. 2014;369(1645):20130433.

2. Moyle PM, Toth I. Modern subunit vaccines: development, components, and research opportunities. ChemMedChem. 2013;8(3): 360-76.

3. Zhao L, Seth A, Wibowo N, Zhao CX, Mitter N, Yu CZ, et al. Nanoparticle vaccines. Vaccine. 2014;32(3):327-37.

4. Reed SG, Orr MT, Fox CB. Key roles of adjuvants in modern vaccines. Nat Med. 2013;19(12):1597-608.

5. De Temmerman ML, Rejman J, Demeester J, Irvine DJ, Gander B, De Smedt SC. Particulate vaccines: on the quest for optimal delivery and immune response. Drug Discov Today. 2011;16(1314):569-82.
6. Fan Y, Moon JJ. Nanoparticle drug delivery systems designed to improve Cancer vaccines and immunotherapy. Vaccines (Basel). 2015;3(3):662-85.

7. Benne N, van Duijn J, Kuiper J, Jiskoot W, Slutter B. Orchestrating immune responses: how size, shape and rigidity affect the immunogenicity of particulate vaccines. J Control Release. 2016;234:12434 .

8. Mody KT, Popat A, Mahony D, Cavallaro AS, Yu CZ, Mitter N. Mesoporous silica nanoparticles as antigen carriers and adjuvants for vaccine delivery. Nanoscale. 2013;5(12):5167-79.

9. Mahony D, Cavallaro AS, Stahr F, Mahony TJ, Qiao SZ, Mitter $\mathrm{N}$. Mesoporous silica nanoparticles act as a self-adjuvant for ovalbumin model antigen in mice. Small. 2013;9(18):3138-46.

10. Mody KT, Mahony D, Zhang J, Cavallaro AS, Zhang B, Popat A, et al. Silica vesicles as nanocarriers and adjuvants for generating both antibody and T-cell mediated immune resposes to bovine viral Diarrhoea virus E2 protein. Biomaterials. 2014;35(37):9972-83.

11. Tu J, Du G, Reza Nejadnik M, Monkare J, van der Maaden K, Bomans PHH, Sommerdijk N, Slutter B, Jiskoot W, Bouwstra JA, Kros A. Mesoporous Silica Nanoparticle-Coated Microneedle Arrays for Intradermal Antigen Delivery Pharm Res 2017

12. Preza I, Subaiya S, Harris JB, Ehlman DC, Wannemuehler K, Wallace AS, et al. Acceptance of the Administration of Multiple Injectable Vaccines in a Single Immunization Visit in Albania. J Infect Dis. 2017;216(suppl_1):S146-51.

13. Danchin MH, Costa-Pinto J, Attwell K, Willaby H, Wiley K, Hoq $\mathrm{M}$, et al. Vaccine decision-making begins in pregnancy: correlation between vaccine concerns, intentions and maternal vaccination with subsequent childhood vaccine uptake. Vaccine. 2017;

14. Grimmond T, Good L. Exposure survey of trends in occupational practice (EXPO-S.T.O.P.) 2015: a national survey of sharps injuries and mucocutaneous blood exposures among health care workers in US hospitals. Am J Infect Control. 2017;45(11):121823.

15. Weiser JR, Saltzman WM. Controlled release for local delivery of drugs: barriers and models. J Control Release : Off J Control Release Soc. 2014;190:664-73.

16. van der Maaden K, Jiskoot W, Bouwstra J. Microneedle technologies for (trans)dermal drug and vaccine delivery. J Control Release. 2012;161(2):645-55.

17. Kim YC, Park JH, Prausnitz MR. Microneedles for drug and vaccine delivery. Adv Drug Deliv Rev. 2012;64(14):1547-68.

18. Leone M, Monkare J, Bouwstra J, Kersten G. Dissolving microneedle patches for dermal vaccination. Pharm Res-Dord. 2017;34(11):2223-40.

19. Koutsonanos DG, del Pilar MM, Zarnitsyn VG, Sullivan SP, Compans RW, Prausnitz MR, et al. Transdermal influenza immunization with vaccine-coated microneedle arrays. PLoS One. 2009;4(3):e4773.

20. van der Maaden K, Yu HX, Sliedregt K, Zwier R, Leboux R, Oguri M, et al. Nanolayered chemical modification of silicon surfaces with ionizable surface groups for $\mathrm{pH}$-triggered protein adsorption and release: application to microneedles. J Mater Chem B. 2013;1(35):4466-77.

21. van der Maaden K, Varypataki EM, Romeijn S, Ossendorp F, Jiskoot W, Bouwstra J. Ovalbumin-coated $\mathrm{pH}$-sensitive microneedle arrays effectively induce ovalbumin-specific antibody and T-cell responses in mice. Eur J Pharm Biopharm. 2014;88(2): $310-5$.

22. van der Maaden K, Sekerdag E, Schipper P, Kersten G, Jiskoot W, Bouwstra J. Layer-by-layer assembly of inactivated poliovirus and $\mathrm{N}$-Trimethyl chitosan on $\mathrm{pH}$-sensitive microneedles for dermal vaccination. Langmuir : ACS J Surfaces Colloid. 2015;31(31):8654 60 .

23. Schipper P, van der Maaden K, Groeneveld V, Ruigrok M, Romeijn S, Uleman S, et al. Diphtheria toxoid and N-trimethyl 
chitosan layer-by-layer coated $\mathrm{pH}$-sensitive microneedles induce potent immune responses upon dermal vaccination in mice. $\mathrm{J}$ Control Release : OffJ Control Release Soc. 2017;262:28-36.

24. Du G, Hathout RM, Nasr M, Nejadnik MR, Tu J, Koning RI, et al. Intradermal vaccination with hollow microneedles: a comparative study of various protein antigen and adjuvant encapsulated nanoparticles. J Control Release : Off J Control Release Soc. 2017;266:109-18.

25. Bal SM, Slutter B, van Riet E, Kruithof AC, Ding Z, Kersten GFA, et al. Efficient induction of immune responses through intradermal vaccination with $\mathrm{N}$-trimethyl chitosan containing antigen formulations. J Control Release. 2010;142(3):374-83.

26. Tu J, Boyle AL, Friedrich H, Bomans PH, Bussmann J, Sommerdijk NA, et al. Mesoporous silica nanoparticles with large pores for the encapsulation and release of proteins. ACS Appl Mater Interfaces. 2016;8(47):32211-9.

27. van der Maaden K, Sekerdag E, Jiskoot W, Bouwstra J. Impactinsertion applicator improves reliability of skin penetration by solid microneedle arrays. AAPS J. 2014;16(4):681-4.

28. van der Maaden K, Heuts J, Camps M, Pontier M, Terwisscha van Scheltinga A, Jiskoot W, et al. Hollow microneedle-mediated micro-injections of a liposomal HPV E743-63 synthetic long peptide vaccine for efficient induction of cytotoxic and T-helper responses. J Control Release : OffJ Control Release Soc. 2018;269:347-54.

29. van der Maaden K, Trietsch SJ, Kraan H, Varypataki EM, Romeijn S, Zwier R, et al. Novel hollow microneedle Technology for Depth-Controlled Microinjection-Mediated Dermal Vaccination: a study with polio vaccine in rats. Pharm Res-Dord. 2014;31(7):1846-54.

30. Amidi M, Pellikaan HC, Hirschberg H, de Boerd AH, Crommelin DJA, Hennink WE, et al. Diphtheria toxoid-containing microparticulate powder formulations for pulmonary vaccination: preparation, characterization and evaluation in Guinea pigs. Vaccine. 2007;25(37-38):6818-29.

31. Ding Z, Van Riet E, Romeijn S, Kersten GFA, Jiskoot W, Bouwstra JA. Immune modulation by adjuvants combined with diphtheria toxoid administered topically in BALB/c mice after microneedle Array pretreatment. Pharm Res-Dord. 2009;26(7):1635-43.

32. Larraneta E, McCrudden MTG, Courtenay AJ, Donnelly RF. Microneedles: a new frontier in nanomedicine delivery. Pharm Res-Dordr. 2016;33(5):1055-73.

33. Li N, Peng LH, Chen X, Nakagawa S, Gao JQ. Transcutaneous vaccines: novel advances in technology and delivery for overcoming the barriers. Vaccine. 2011;29(37):6179-90.

34. Peek LJ, Middaugh CR, Berkland C. Nanotechnology in vaccine delivery. Adv Drug Deliv Rev. 2008;60(8):915-28.

35. Mourya VK, Inamdar NN. Trimethyl chitosan and its applications in drug delivery. J Mater Sci Mater Med. 2009;20(5):1057-79.

36. DeMuth PC, Moon JJ, Suh H, Hammond PT, Irvine DJ. Releasable layer-by-layer assembly of stabilized lipid nanocapsules on microneedles for enhanced transcutaneous vaccine delivery. ACS Nano. 2012;6(9):8041-51.

37. Bronaugh RL, Stewart RF, Congdon ER. Methods for in vitro percutaneous absorption studies. II. Animal models for human skin. Toxicol Appl Pharmacol. 1982;62(3):481-8.

38. Slutter B, Bal SM, Ding Z, Jiskoot W, Bouwstra JA. Adjuvant effect of cationic liposomes and $\mathrm{CpG}$ depends on administration route. J Control Release : Off Journal Control Release Soc. 2011;154(2): 123-30.

39. Bal SM, Ding Z, Kersten GFA, Jiskoot W, Bouwstra JA. Microneedle-based transcutaneous immunisation in mice with $\mathrm{N}$ Trimethyl chitosan Adjuvanted diphtheria toxoid formulations. Pharm Res-Dord. 2010;27(9):1837-47. 